Portland State University

PDXScholar

\title{
Discrete Sums for the Rapid Determination of Exponential Decay Constants
}

\author{
Michael A. Everest \\ George Fox University \\ Dean B. Atkinson \\ Portland State University, atkinsond@pdx.edu
}

Follow this and additional works at: https://pdxscholar.library.pdx.edu/chem_fac

Part of the Chemistry Commons

Let us know how access to this document benefits you.

\section{Citation Details}

Everest, M. A., \& Atkinson, D. B. (2008). Discrete sums for the rapid determination of exponential decay constants. Review Of Scientific Instruments, 79(2), 023108.

This Article is brought to you for free and open access. It has been accepted for inclusion in Chemistry Faculty Publications and Presentations by an authorized administrator of PDXScholar. Please contact us if we can make this document more accessible: pdxscholar@pdx.edu. 


\title{
Discrete sums for the rapid determination of exponential decay constants
}

\author{
Michael A. Everest ${ }^{1, a)}$ and Dean B. Atkinson ${ }^{2}$ \\ ${ }^{1}$ Department of Biology and Chemistry, George Fox University, 414 N Meridian St., Newberg, Oregon \\ 97132, USA \\ ${ }^{2}$ Department of Chemistry, Portland State University, P.O. Box 751, Portland, Oregon 97201-0751, USA
}

(Received 9 November 2007; accepted 14 January 2008; published online 28 February 2008)

\begin{abstract}
Several computational methods are presented for the rapid extraction of decay time constants from discrete exponential data. Two methods are found to be comparably fast and highly accurate. They are corrected successive integration and a method involving the Fourier transform (FT) of the data and the application of an expression that does not assume continuous data. FT methods in the literature are found to introduce significant systematic error owing to the assumption that data are continuous. Corrected successive integration methods in the literature are correct, but we offer a more direct way of applying them which we call linear regression of the sum. We recommend the use of the latter over FT-based methods, as the FT methods are more affected by noise in the original data. () 2008 American Institute of Physics. [DOI: 10.1063/1.2839918]
\end{abstract}

\section{INTRODUCTION}

Several fields of scientific inquiry rely on the extraction of the time constant from data that decay exponentially. Examples include fluorescence lifetimes, nuclear decay, firstorder chemical kinetics, and cavity ring-down (CRD) spectroscopy. Frequently, the data are acquired on a time scale sufficiently long to permit the iterative fitting of the data to an exponential functional form using an algorithm such as the nonlinear Levenberg-Marquardt algorithm. However, in recent years some experiments have advanced to the point where the fitting of the data is the slowest step in the data acquisition process. In these cases, faster methods of extracting exponential decay constants will increase the rate at which the overall experiment can be conducted.

\section{A. Fourier transform}

To date, two significantly faster computational methods have appeared in the literature. Kirchner et al. applied the Fourier transform to transients in deep-level transient spectroscopy to extract the exponential decay constant. ${ }^{1}$ Specifically, they showed that if the data decay according to

$$
f(t)=A e^{-\beta t}+b,
$$

then the decay time constant can be determined from the Fourier transform,

$$
F(\omega)=\int_{0}^{\infty} f(t) e^{i \omega t} \mathrm{~d} t
$$

from the following relation:

$$
\beta=\omega \frac{\operatorname{Re}[F(\omega)]}{\operatorname{Im}[F(\omega)]} .
$$

The derivation of Eq. (3) is included in the Appendix.

\footnotetext{
${ }^{\text {a)} E l e c t r o n i c ~ m a i l: ~ m e v e r e s t @ g e o r g e f o x . e d u . ~}$
}

Interest in using the Fourier transform to extract exponential decay constants was recently renewed by Mazurenka et al. who used Kirchner's method to analyze data from cavity ring-down spectroscopy. ${ }^{2}$ (Table I includes abbreviations and a short description of all the computational methods discussed in this paper.) They found that using the fast Fourier transform (FFT) and Eq. (3) is faster than the LevenbergMarquardt iterative fitting, and nearly as accurate. Since their publication, several other published studies have also employed this method. ${ }^{3-6}$

As can be seen from Eq. (3), the decay constant $\beta$ should be the same, regardless of which frequency component of the Fourier transform is used. In practice, the lowest frequency component of the Fast Fourier transform has been chosen. However, if $\beta$ is estimated according to the method of Kirchner et al., it is in fact found to be frequency dependent as can be seen by the dashed trace in Fig. 1. This curve was calculated from the FFT of a 1000-point exponentially decaying waveform with a ring-down time constant $\tau=1 / \beta=1.5 \mu \mathrm{s}$ extending for $9 \mu \mathrm{s}$. The value of $\tau$ estimated at the lowest frequency component $(111.111 \mathrm{kHz})$ is $1.49998 \mu \mathrm{s}$.

In order to estimate the decay constant, Kirchner et al. warn that "one must exercise caution that the frequency components introduced by the discontinuity from $f(0)$ to $f\left(t_{m}\right)$ are small with respect to those from the exponential. Typically, this can be accomplished by making the first sample equal to the average of the first and last samples." ${ }^{11}$ That is, before taking the FFT of the raw data, the value in the data at $t=0$ must be replaced with the average between the first and last points of the exponentially decaying waveform.

The dotted curve in Fig. 1 was also calculated from Eq. (3), but without initially replacing the first point as described in the preceding paragraph. Clearly, neglecting this step leads to significant error in the estimation of the decay time. The value of $\tau$ at the lowest frequency component is $1.49061 \mu \mathrm{s}$, a systematic error of nearly $1 \%$. Mazurenka et al. report obtaining a decay time of $32.49 \pm 0.01 \mu$ s from a 15000 - 
TABLE I. Abbreviations and brief descriptions of algorithms discussed in the text.

\begin{tabular}{ll}
\hline \hline \multicolumn{1}{c}{ Method } & \multicolumn{1}{c}{ Description } \\
\hline LM & $\begin{array}{l}\text { Levenberg-Marquardt } \\
\text { FFT-FPR }\end{array}$ \\
& $\begin{array}{l}\text { First-point replacement (FRP) in raw data, FFT, } \\
\text { then Eq. (3) is used with the first frequency } \\
\text { component (Ref. 1). } \\
\text { No first-point replacement in raw data, FFT, } \\
\text { then Eq. (3) with the first frequency component (Ref. 9). }\end{array}$ \\
FFT-NFPR & $\begin{array}{l}\text { FFT then Eq. (9) with the first frequency component. } \\
\text { (This work.) }\end{array}$ \\
DFT-5 & $\begin{array}{l}\text { FFT then Eq. (9) with weighted average of first } \\
\text { five frequency components. (This work.) } \\
\text { Trapezoidal integration, solution of linear }\end{array}$ \\
CSI & $\begin{array}{l}\text { least squares, approximate value for } \tau \text {, then } \\
\text { a correction in Eq. (8) (Ref. 7). }\end{array}$ \\
Rectangular integration, solution of linear \\
LRS
\end{tabular}

point exponentially decaying waveform with a decay time of $32.5 \mu \mathrm{s} .^{2}$ We are able to reproduce that value by neglecting to replace the first point of the decay curve before taking the FFT, which leads us to believe that they did not perform the first-point replacement in their study.

Kirchner et al. included a negative sign in the expression for $\beta$ in Eq. (3). The reason for this difference is that there is no consensus as to whether the FT has a $-i \omega t$ or a $+i \omega t$ in the exponential of Eq. (2). This is equivalent to exchanging definitions of the FT and the inverse FT. A brief survey shows that some textbooks and commercial software use $i \omega t$ for the FT (IGORPRO), and other software packages use $-i \omega t$ (LABVIEW).

\section{B. Corrected successive integration}

A second method for the rapid determination of $\beta$ from experimental data is the method of corrected successive integration (CSI). ${ }^{7,8}$ This method relies on the fact that the integral of an exponentially decaying waveform also has an

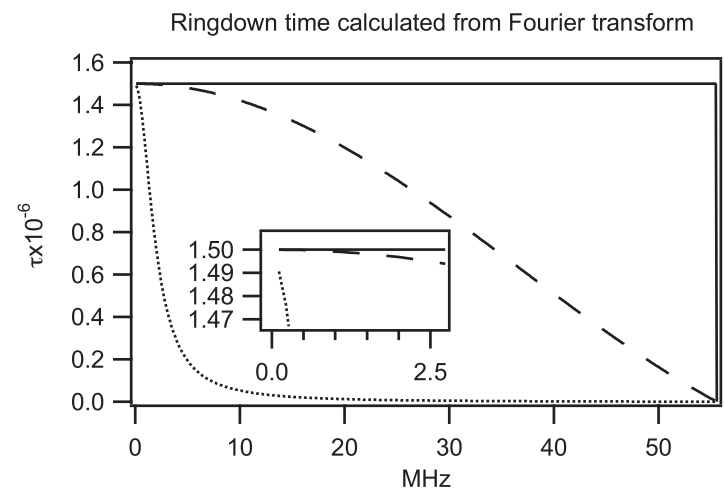

FIG. 1. The Fourier-transform extracted exponential decay time constant as a function of frequency. The dashed curve is calculated according to Kirchner et al. (Ref. 1), the dotted curve is calculated according to Mazurenka et al. (Ref. 2), and the solid curve is calculated according to the procedure (DFT-1) described in the text. The inset shows the behavior as the curves approach the low-frequency limit. exponential component. If the waveform of interest follows Eq. (1), then the original waveform may be written as a function of its own integral,

$$
\begin{aligned}
& \int_{0}^{t} f(t) \mathrm{d} t=\frac{A+b}{\beta}-\frac{1}{\beta} f(t)+b t, \\
& f(t)=A+b-\beta \int_{0}^{t} f(t) \mathrm{d} t+\beta b t .
\end{aligned}
$$

This equation is of the form $y\left(x_{1}, x_{2}\right)=a_{0}+a_{1} x_{1}+a_{2} x_{2}$ where the two independent variables are the integral in the second term and $t$ in the third. Least-squares fitting can be used to determine the coefficients of this equation, and consequently $A, b$, and $\beta$.

This fitting is accomplished by solving the following matrix equation for $a_{0}, a_{1}$, and $a_{2}$ :

$$
\left[\begin{array}{lll}
N & S_{I} & S_{t} \\
S_{I} & S_{I I} & S_{t I} \\
S_{t} & S_{t I} & S_{t t}
\end{array}\right]\left[\begin{array}{l}
a_{0} \\
a_{1} \\
a_{2}
\end{array}\right]=\left[\begin{array}{c}
S_{f} \\
S_{f I} \\
S_{f t}
\end{array}\right] .
$$

In addition to the number of data points, $N$, and the fitting coefficients $a_{0}, a_{1}$, and $a_{2}$, this equation contains two types of sums $S$ : sums over individual values and sums over the product of two values. The type of sum is indicated by the number of subscripts,

$$
\begin{aligned}
S_{g} & \equiv \sum_{i=0}^{N-1} g_{i}, \\
S_{g h} & \equiv \sum_{i=0}^{N-1} g_{i} h_{i} .
\end{aligned}
$$

The terms in these sums are the data values in the original waveform $f_{i}$, the time at which these values occur $t_{i}$, and the running integral of the data,

$$
I_{i} \equiv \int_{0}^{t_{i}} f(t) \mathrm{d} t .
$$

In practice, the running integral $I$ is determined first, then each sum in the matrices in Eq. (6) is calculated, the matrix equation is then solved for $a_{0}, a_{1}$, and $a_{2}$ from which the original constants in the exponential function $(A, b$, and $\beta)$ may be determined.

To improve computational speed Halmer et al. ${ }^{7}$ evaluate the sums over $t$ directly,

$$
\begin{aligned}
& S_{t}=\frac{N(N+1)}{2}, \\
& S_{t t}=\frac{N(N+1)(2 N+1)}{6} .
\end{aligned}
$$

While Halmer et al. define $N$ to be the index of their last point, we define $N$ as the total number of points. Therefore, we put $N-1$ for each occurrence of $N$ in the previous expression.

Real data are, of course, discrete, so the integral in Eq. (7) must be evaluated as a sum. Matheson found trapezoidal integration to be a sufficient approximation of the integral, ${ }^{8}$ 
but Halmer et al. found an additional correction to be required for their data. ${ }^{7}$ Having first determined an approximate value of the decay constant from the above procedure, they eliminated the error introduced by the trapezoidal integration as follows:

$$
\tilde{\tau}=\frac{1}{\ln [(2 \tau+1) /(2 \tau-1)]},
$$

in which $\tau$ is the initial approximate value, and $\widetilde{\tau}$ is the corrected value. This corrected method has been applied in several studies. ${ }^{9-22}$

\section{Current applications to CRD spectroscopy}

The most rapid data acquisition and throughput for a ring-down experiment is the purely analog method pioneered by Spence et al. at Stanford University. ${ }^{23}$ Unfortunately, this approach is more experimentally demanding than the traditional method that involves digitizing or otherwise recording the ring-down decay and then extracting the decay constant. (There is also a chance that the extraction of the decay constant in the analog approach can suffer from drift, if all of the components are not well matched and temperature compensated.) Most of the CRD instruments being used in practical applications capture a time-intensity decay signature and extract the decay rate using mathematical procedures such as those detailed here.

There are a number of reports in the literature of cavity ring-down applications that benefit from rapid data fitting like that documented in this report. Most of these applications implement the cw-CRD approach which can often result in ring-down acquisition rates that are higher than the typical repetition rate of pulsed lasers. An excellent example is the recently reported optical feedback cw-CRD method that uses the light exiting the cavity to seed a diode laser resulting in ring-down initiation/collection rates that can be in the high kilohertz. ${ }^{24,25}$ We will use a multichannel pulsed laser application that results in a fairly high cavity ring-down throughput rate as a demonstration of the power of these data reduction approaches. This instrument, developed under NOAA support for the measurement of aerosol optical properties, contains 12 separate ring-down cavities (four each at 355,532 , and $1064 \mathrm{~nm}$ ) all charged by the same Nd:YAG laser operating at $15 \mathrm{~Hz}$. The effective ring-down acquisition rate for this instrument eventually will be $12 \times 15 \mathrm{~Hz}$ $=180 \mathrm{~Hz}$, although we only used eight channels for this demonstration study, resulting in a $120 \mathrm{~Hz}$ throughput. This high rate is a substantial challenge even for well-coded Levenberg-Marquardt (LM) nonlinear fitting routines. As several authors have noted, the NI LABVIEW implementation of LM is a user friendly virtual instrument (VI), but is not very efficient computationally.

\section{DISCRETE SUMS}

\section{A. Fourier transform}

The errors evident in Fig. 1 arise because Eq. (3) was derived assuming continuous data [i.e., the use of the integral in Eq. (2)]. However, actual data are nearly always discrete, not continuous. To determine a correct expression for the decay constant from discrete data, we must take the discrete Fourier transform (DFT) or the mathematically equivalent but much more rapid FFT of an exponentially decaying waveform, $f_{n}=e^{-\beta n \Delta t}$, where $n$ is the index on the discrete points and goes from 0 to $N-1$, and $\Delta t$ is the separation in time between subsequent data points.

In Appendix $\mathrm{B}$, the following expression for $\beta$ is determined starting with the sum in the DFT rather than the integral in Eq. (2):

$$
\beta=\frac{1}{\Delta t} \ln \left\{\frac{\operatorname{Re}\left[F\left(\omega_{k}\right)\right]}{\operatorname{Im}\left[F\left(\omega_{k}\right)\right]} \sin \omega_{k} \Delta t+\cos \omega_{k} \Delta t\right\},
$$

where $k$ is the index on the frequency and $\omega_{k}=2 \pi k / N \Delta t$. This equation approaches Eq. (3) as $\Delta t \rightarrow 0$.

The solid curve in Fig. 1 was calculated from Eq. (9). The value of $\tau$ calculated using Eq. (9) is exactly equal to $1.5 \mu \mathrm{s}$ for all $\omega_{k}(1 \leqslant k \leqslant 500)$. We therefore conclude that the error in the dashed curve in Fig. 1 is entirely owing to the fact that Eq. (3) was derived assuming continuous data [i.e., the use of the integral in Eq. (2)]. This expression does not require that the first point in the data be replaced before the FFT.

\section{B. Corrected successive integration}

The method of corrected successive integration may also be reexamined in light of using a discrete sum in place of an integral. Instead of using trapezoidal integration and then correcting the value using Eq. (8), as was done by Halmer et al., ${ }^{7}$ the integral in Eq. (7) can be treated as a sum from the beginning.

In this treatment of the data, the direct sum is used instead of trapezoidal integration in the evaluation of $I_{i}$. Following the solution of Eq. (6), $\beta$ is found from $a_{1}$ according to

$$
\beta=\frac{1}{\Delta t} \ln \left(1-a_{1} \Delta t\right) .
$$

This equation converges on the continuous case (i.e., $\left.\beta=-a_{1}\right)$ as $\Delta t \rightarrow 0$. A derivation of Eq. (10) is in Appendix C.

Because this method does not have any necessary connection with the integral, we will call it linear regression of the sum (LRS) from here forward.

Although the algorithms are different, both LRS and the method used by Halmer et al. are exact and give accurate results when applied to simulated data. Halmer et al. use an approximate solution and then apply a correction, ${ }^{7}$ while the present method permits the direct evaluation of $\beta$ without a correction.

\section{COMPARISON OF METHODS}

\section{A. Speed}

The computation time for the various methods is shown in Fig. 2 as it depends on the total number of data points in the exponential waveform. In general, DFT-1 and LRS are roughly an order of magnitude faster than the LM. However, at certain values of $N$, specifically when $N$ is highly factorizable, DFT-1 may be faster than LRS. This is shown in the inset of Fig. 2 which shows that the time to run DFT-1 at 
Fit Time Dependence on $N$

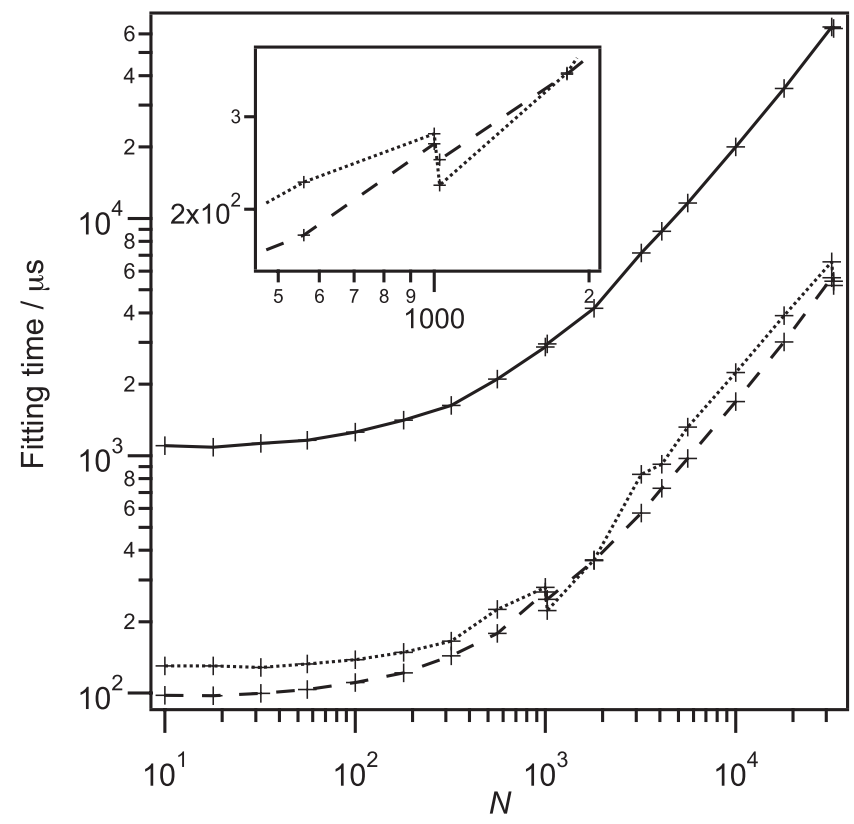

FIG. 2. The dependence of the fitting time on the number of data points in the exponential waveform. The solid curve is for the Levenberg-Marquardt, the dotted curve is calculated according to the DFT algorithm, and the dashed curve is calculated according to the linear regression of the sum. The inset shows the nonmonotonic behavior of the DFT algorithm at highly factorizable values of $N$.

$N=1024$ is significantly less than at $N=1000$. This is not always the case. The LRS algorithm is slightly faster than DFT- 1 at $N=4096$.

The exact speed of the calculations will likely depend significantly on the computational platform and software used. A highly optimized DFT-1 algorithm may very well outperform a poorly coded LRS. It will generally be true that both of these noniterative methods will always be significantly faster than the LM.

Because only one frequency component is needed for the DFT-1 algorithm, it is not necessary to perform the FFT of the waveform. The sum in Eq. (B1) of the the Appendix can be performed directly for the single value $k=1$. In our implementation, this was not faster than the FFT available in our commercial software package - an indication of the remarkable efficiency of the FFT algorithm.

It should also be noted that the actual FFT of the waveform is not required, only the ratio of the real to the imaginary component for one frequency. As is shown in Appendix $\mathrm{D}$, this is equivalent to knowing only the phase of the FFT at this frequency. Although calculating only the phase of the FFT was no faster in our implementation, we mention this possibility because it may be faster on other computational platforms. Moreover, it may be possible to determine the phase of a particular Fourier component with analog electronic hardware, preventing the need for rapid digitization of the entire waveform. The use of the phase shift to measure the effective loss in an optical cavity has already been demonstrated by Engeln et al. for a modulated CW source. ${ }^{26}$
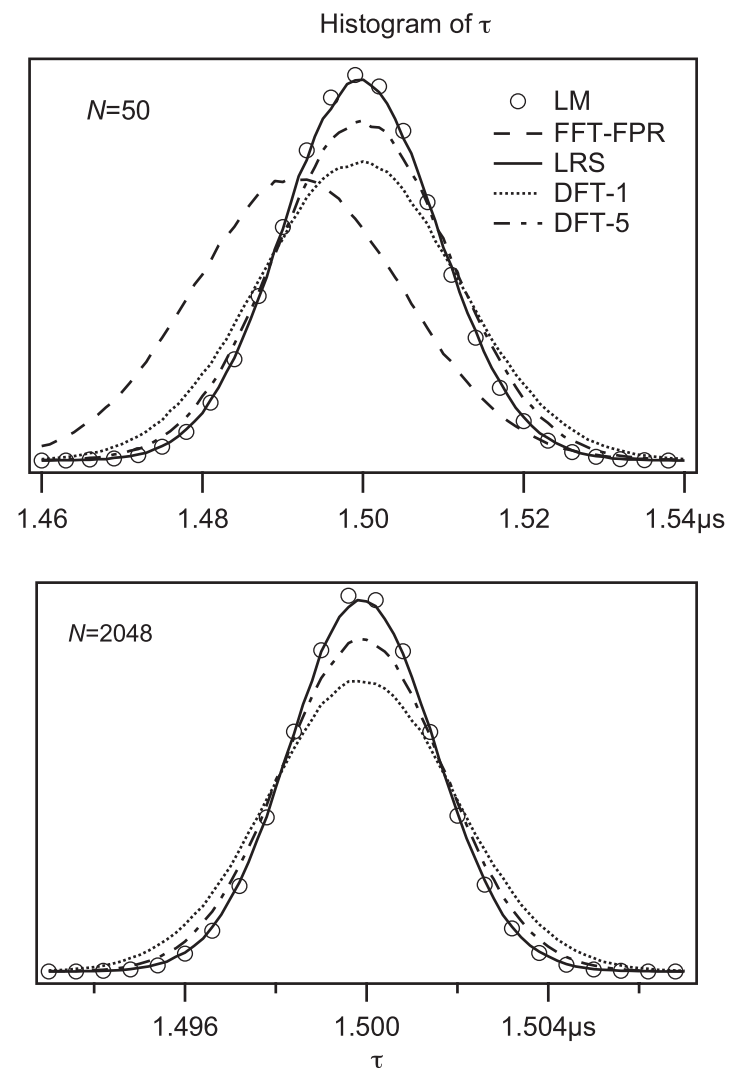

FIG. 3. Histograms of $\tau$ evaluated from noisy simulated data using various algorithms. The upper figure is for data sets with 50 points, the lower figure for data sets 2048 points. Note the different $x$ axes. For all curves the noise level is $1 \%$, the true value of $\tau$ is $1.5 \mu \mathrm{s}$, and the total length of the data waveform is $9 \mu \mathrm{s}$. The curve for FFT-FPR is not shown in the lower figure as it would obscure the curve for DFT-1.

\section{B. Accuracy and precision}

Figure 3 shows a histogram of the value of $\tau$ recovered for several different algorithms and two different numbers of data points. The top figure is for $N=50$ and the lower figure is for $N=2048$. 500000 data sets were simulated with $\tau=1.5 \mu \mathrm{s}$, a total waveform length $\left(t_{m}\right)$ of $9 \mu \mathrm{s}$, and a noise level of $1 \%$. The LRS, DFT-1, and LM all give results that are very accurate, that is, the correct value for $\tau$ is recovered from the average of many data sets. The histogram of $\tau$ 's evaluated from the FFT expression that assumes that the data are continuous (i.e., FFT-FPR) is not centered on $\tau=1.5 \mu$ s, indicating systematic error in this algorithm. Specifically, the value of $\tau$ recovered from the data is systematically too low.

There is also a noticeable difference in the width of the histograms in Fig. 3. This indicates differing precision, or noise immunity, of the various algorithms. The LM and the LRS are found to be the most immune to noise in the data, while the FT methods are slightly broader.

The dependence of this scatter on the total record length is shown in Fig. 4. All curves were generated by finding the standard deviation of 10000 estimates of $\tau$ from data sets having 1000 points, $1 \%$ noise, and $\tau=1.5 \mu$ s. All methods go through a minimum in the spread of the data, but they do so at slightly different record lengths. The FT methods perform the best for total record lengths of $(4-5) \tau$, while the least- 
Dependence of $\sigma_{\tau}$ on $t_{m}$

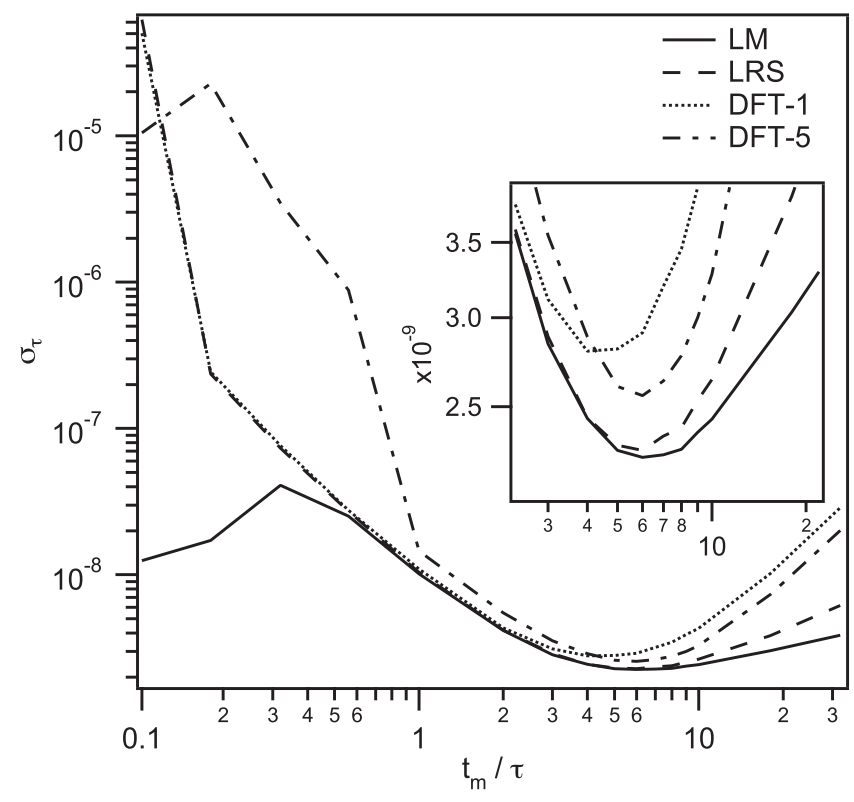

FIG. 4. The standard deviation 10000 separate estimates of $\tau$ from 1000point waveforms of varying length. The noise level is $1 \%$. The inset shows the minimum of the curves in detail. Note that different methods have different optimal record lengths.

squares methods perform the best for record lengths of approximately $(5-7) \tau$.

Table II shows the average $\tau$ and the standard deviation for 10000 separate determinations of $\tau$ for 10000 -point raw data waveforms with $1 \%$ noise and $t_{m}=9 \mu \mathrm{s}$. All the FT methods discussed thus far have a standard deviation of approximately $30 \%$ greater than methods based on least squares, whether iterative (LM) or direct (CSI or LRS). This was found to be true for noise levels ranging from $\left(1 \times 10^{-5}\right) \%$ to $100 \%$.

We suspect that the FT methods demonstrate slightly more scatter because they spread the information about $\tau$ over multiple frequency components in the FFT of the data, but only one frequency component is used in the subsequent determination of $\tau$. Slightly higher noise immunity can be accomplished by estimating $\tau$ from a weighted average of several frequency components of the FFT of the data. Weighting coefficients used were one over the the variance of $\tau$ estimated from 10000 simulated data sets with $\tau=1.5 \mu \mathrm{s}, t_{m}=9 \mu \mathrm{s}, N=1000$, and $1 \%$ noise. The weighting

TABLE II. The average and standard deviation of $\tau$ recovered from various fitting methods. 10000 separate 10000 -point raw data waveforms with $1 \%$ noise were analyzed.

\begin{tabular}{cc}
\hline \hline Method & Recovered $\tau / \mu \mathrm{s}$ \\
\hline LM & $1.49999 \pm(71)$ \\
FFT-FPR & $1.50000 \pm(94)$ \\
FFT-NFPR & $1.49904 \pm(92)$ \\
DFT-1 & $1.50000 \pm(93)$ \\
DFT-5 & $1.50000 \pm(81)$ \\
CSI & $1.50001 \pm(73)$ \\
LRS & $1.50000 \pm(72)$ \\
\hline \hline
\end{tabular}

TABLE III. Weighting coefficients for the frequency components for the evaluation of $\tau$ from a weighted average of the first five frequency components of the FFT.

\begin{tabular}{ll}
\hline \hline$k$ & Weight \\
\hline 1 & 1.0 \\
2 & 0.274068 \\
3 & 0.0977867 \\
4 & 0.0427595 \\
5 & 0.0216585 \\
\hline
\end{tabular}

coefficients for the first five frequency components are listed in Table III. As can be seen in Fig. 3, this method (DFT-5) leads to a slight reduction of the spread in $\tau$. This is generally the case for noise levels up to $10 \%$, after which the the standard deviation of $\tau$ becomes much worse than the other methods. As is seen in Table II, at a noise level of $1 \%$ for 10 000-point data, the DFT-5 method had a standard deviation of approximately $0.81 \mathrm{~ns}$.

\section{EXPERIMENT}

The LRS and DFT-1 approaches explained above were coded into a series of LABVIEW VIs (available upon request from the authors). These VIs were installed on a CoreDuo Pentium computer in a NI PXI chassis that also contained an eight-channel high-speed high-density data acquisition device (National Instruments, Inc. PXI-1031, PXI-8105, and PXI-5105). The close coupling of the digitizer and the computer (both on the same PCI bus) allows a rapid enough data transfer rate to accommodate the eight channels of approximately 1500 digitized points each, without becoming the limiting factor in the total ring-down signal throughput. For this demonstration experiment, a single ring-down signal was delivered to all eight channels (one of which was operated at $50 \Omega$ ) to allow the interdigitizer variability to be assessed, in addition to the speed of the data reduction procedures.

The ring-down signal was generated using a single channel of our new humidity controlled cavity ring-down transmissometer/nephelometer (HC-CRDT/N) instrument. Briefly, the visible $(532 \mathrm{~nm})$ beam from an Nd:YAG laser (Big Sky Laser, Inc. CFR200-15 Ultra) operating at $15 \mathrm{~Hz}$ is coupled into a multifiber bundle (Ceramoptec Industries, Inc.) which separates into four smaller bundles, one of which delivers the light (typically $<1 \mathrm{~mJ}$ ) to a single ring-down cavity. The light exiting the fiber bundle is approximately collimated by a two-lens system and launched into the ringdown cavity. The ring-down cavity consists of two $7.75 \mathrm{~mm}$ diameter, $-1 \mathrm{~m}$ radius of curvature superpolished mirrors coated for maximum reflectivity at $532 \mathrm{~nm}$ (Layertec LLC, Mainz, DDR) mounted $96 \mathrm{~cm}$ apart in custom mounts. An end-on Hamamatsu photomultiplier assembly operated at $-500 \mathrm{~V}_{\mathrm{DC}}$ is used to detect the light after it exits the cavity with no other coupling optics besides a $532 \mathrm{~nm}$ bandpass filter.

Interestingly, there is no evidence of the typically observed transverse mode beating structure on the ring-down signals, possibly because of the lack of spatial variability in 
Ringdown Data

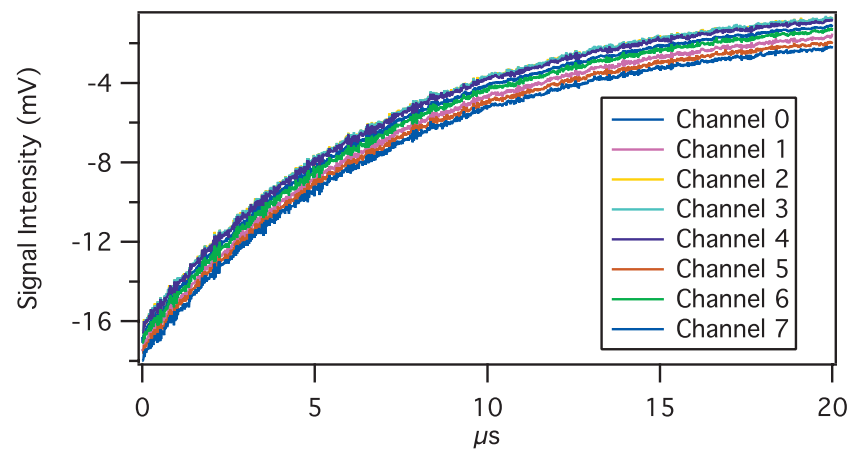

FIG. 5. (Color) An example of the ring-down data used to test the speed and quality of the two high-speed data reduction techniques detailed in this paper. A single ring-down signal was digitized by eight separate ADCs within a single acquisition card at $60 \mathrm{MSa} / \mathrm{s}$.

conversion efficiency on the photocathode of the end-on PMT. We also note that the longitudinal mode beating structure, that is expected because the bandwidth of the Big Sky laser $\left(<2.0 \mathrm{~cm}^{-1}\right.$ at $\left.532 \mathrm{~nm}\right)$ exceeds the $\approx 500 \mathrm{MHz}$ longitudinal mode spacing, is suppressed. This suppression is probably due to a combination of the somewhat longer pulse widths (12-15 ns specified for the CFR200 versus the 3-5 ns for many nanosecond pulsed lasers) and the $60 \mathrm{MHz}$ analog input filter on the NI digitizer used.

\section{RESULTS}

As explained above, a single ring-down signal with a time constant of about $6 \mu$ s was delivered to the eight channels of the digitizer in parallel. An example of a single-laserpulse set of eight ring-down signals is shown in Fig. 5. As noted above, there is little evidence of the low-frequency mode beating structure that is typically observed on ringdown signals. The initial spike and region of nonexponential decay (about $2 \mu \mathrm{s}$ ) was truncated from the raw signals, as verified by examining the residuals to the LRS fit procedure (not shown here.) It is interesting to note that there is a slight vertical offset between the individual channels (with a spread of the order of $2 \mathrm{mV}$ )—surprising because the signal source is the same for all eight. The values represented here are voltages, rather than raw analog to digital converter (ADC) output, so calibration drift could be the source of offset. Since the desired information content of a ring-down signal is the relative intensity change per unit time, this constant offset should not be a problem in practical CRD measurements.

The digitized signals were then subjected to fitting by the NI LABVIEW VIs that carried out the DFT-1 and LRS fitting procedures, and both procedures were found able to obtain the ring-down parameters in real time at the $15 \mathrm{~Hz}$ repetition rate of the YAG laser. Timing monitors within the fitting procedures showed that the time between fits is always less than or equal to $67 \mathrm{~ms}(1 / 15 \mathrm{~Hz})$ implying that the laser repetition rate limits the ring-down acquisition rate, not the analog to digital conversion, data transfer, or data reduction portions of the procedure. The total cavity loss, expressed in units of inverse megameters $\mathrm{Mm}^{-1}[c / \beta$, where $c$ is the
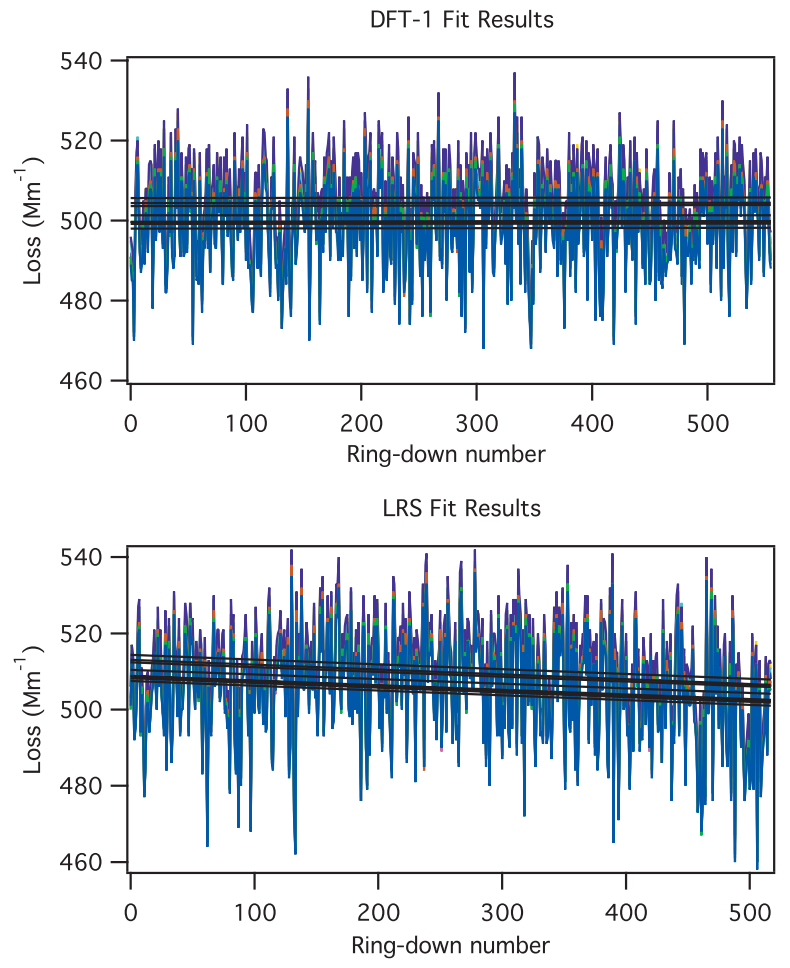

FIG. 6. (Color) The results of real-time acquisition and fitting of the eightchannel ring-down signals over the course of about 500 laser shots $(\approx 30 \mathrm{~s})$. The DFT-1 routine is the top panel, while the LRS procedure is the lower panel. The fits are not to the same 500 laser shots. The solid lines in each figure are linear fits to the time dependent ring-down loss data.

speed of light and $\beta$ is the time-based decay constant from Eq. (1)] for about 500 individual ring-down events is shown in Fig. 6 for the DFT-1 and LRS procedure, respectively. (The $\mathrm{Mm}^{-1}$ units are commonly used in aerosol optics and are essentially equal to the parts-per-million per pass unit often used in the CRD literature, since our cavity is almost exactly one meter long.) Unfortunately, during the collection of the LRS data, the losses in the nonevacuated instrument appear to have shifted a bit resulting in a downward trend in the data that is accentuated by the linear fits (solid black lines) in the figure.

Despite this problem, a number of observations can be made about the data: (1) the mean ring-down loss obtained by the two fitting routines is essentially the same, within the uncertainty imposed by the clearly drifting cavity losses, (2) the results from some channels are offset from those of the others, although the spread between channels is significantly smaller than the spread in the time variation of the individual ring downs, and (3) the channels that give higher results do so consistently across the trend and between methods. These last two observations may be tied to the aforementioned possibility of calibration drift in the ADCs, a possibility that we will investigate in the future. Fortunately, the conclusion from the last observation is that the (relatively small and consistent) interchannel differences in cavity loss is not a failing of the data reduction procedures that are the subject of this work.

To verify that the ring-down losses calculated by the two high-speed data reduction procedures are normally distributed and can thus be used in average to provide a lower 

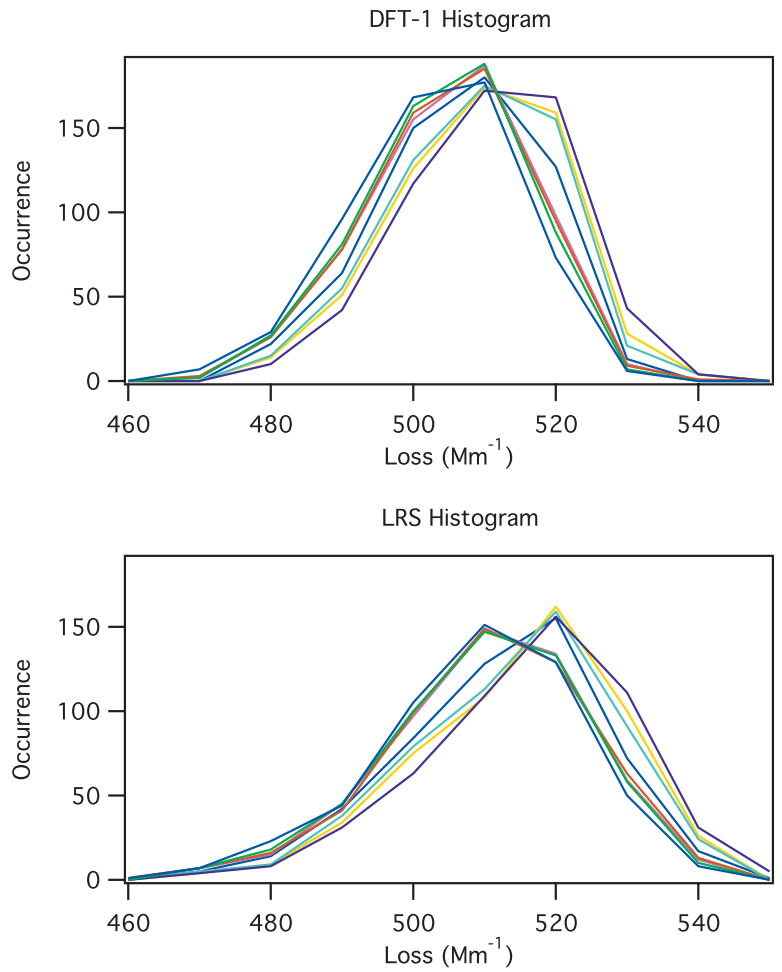

FIG. 7. (Color) The histograms of the results presented in Fig. 6. The top panel is for the DFT-1 data reduction procedure, while the bottom panel is the LRS method. Clearly, both obtain the same result, within error and the long-term drift of the system noted above. Both also clearly produce normally distributed loss data that can be expected to improve with signal averaging.

uncertainty result, we created histograms of the results in Fig. 6, as shown in Fig. 7. The systematic difference in ringdown losses from the different channels can also be seen as a shift in the distributions, for example, between channel 1 and channel 4. Again, because it is the difference in the decay rates (or cavity losses) between a base case (e.g., an evacuated cavity) and the measurement condition that is related to optical extinction in practical ring-down experiments, it is possible that these otherwise disquieting observations may be unimportant.

\section{CONCLUSION}

Of the two types of rapid algorithms considered, both FT methods and methods based on linear regression of the direct sum are equally fast and accurate. The former has the advantage of being significantly easier to implement, but has the disadvantage of being slightly more susceptible to noise in the data than the latter.

If the FT is used, we recommend that the FFT be performed on the raw data and that Eq. (9) be used to extract the exponential time constant (although Eq. (3) also gives fairly accurate results if the first point in the exponential waveform is replaced by the average of the first and last points before the FFT is performed). The noise immunity of the FFT algorithm can be improved by taking the weighted average of multiple frequency points; however, we do not recommend this as the influence of the noise can be unexpected and catastrophic, as can be seen in Fig. 4 at low values of $t_{m}$ for the DFT-5 curve.

If one requires a fast algorithm with the minimum scatter in the data, we recommend that the direct sum (corresponding to rectangular numerical integration) of the data be used, followed by the solution of Eq. (6), and that Eq. (10) be used to obtain $\tau$. This algorithm gives results that are equivalent to using trapezoidal integration followed by a correction to $\tau$ from Eq. (8), but we find the latter to be unnecessarily complex.

The implications of the experimental portion of this study are that the two high-speed exponential decay constant extraction procedures described in this paper are reliable and significantly faster than the LM procedure, making online real-time extraction of ring-down information possible in instruments at throughputs in excess of $100 \mathrm{~Hz}$, provided that the data can be digitized and transferred at a sufficient rate. It is also useful to point out that the data reduction programs were quite easy to code and that the authors are happy to provide the NI LABVIEW VIs or IGORPRO programs to save even that expenditure of effort.

\section{ACKNOWLEDGMENTS}

We thank John Johnson for helpful comments in the preparation of this manuscript. M.A.E. was supported in part by the George Fox University Grant No. GFU07G0004. D.B.A. wishes to acknowledge the support of the NOAA Atmospheric Climate and Composition program through Grant No. NA05OAR4310108.

\section{APPENDIX A: FOURIER TRANSFORM OF $e^{-\beta t}$}

The Fourier transform of $e^{-\beta t}$ and its use in finding $\beta$ is as follows:

$$
\begin{aligned}
F(\omega) & =\int_{0}^{\infty} e^{-\beta t} e^{i \omega t} \mathrm{~d} t \\
& =\int_{0}^{\infty} e^{-(\beta-i \omega) t} \mathrm{~d} t,
\end{aligned}
$$

and with

$\int_{0}^{\infty} e^{-a t} \mathrm{~d} t=\frac{1}{a}$,

we find that

$$
F(\omega)=\frac{1}{\beta-i \omega} .
$$

Multiplying this by the complex conjugate of the denominator,

$$
\begin{aligned}
F(\omega) & =\frac{1}{\beta-i \omega} \cdot \frac{\beta+i \omega}{\beta+i \omega} \\
& =\frac{\beta}{\beta^{2}+\omega^{2}}+i \frac{\omega}{\beta^{2}+\omega^{2}} .
\end{aligned}
$$

From this expression, it is straightforward to show that 


$$
\beta=\omega \frac{\operatorname{Re}[F(\omega)]}{\operatorname{Im}[F(\omega)]}
$$

for all $\omega$.

\section{APPENDIX B: DETERMINATION OF $\boldsymbol{\beta}$ FROM THE DFT}

The DFT of the an exponentially decaying waveform, $f_{n}=e^{-\beta n \Delta t}$, is given by

$$
\begin{aligned}
& F_{k} \equiv \sum_{n=0}^{N-1} f_{n} e^{2 \pi i n k / N} \\
& =\sum_{n=0}^{N-1} e^{-(\beta \Delta t-2 \pi i k / N) n} .
\end{aligned}
$$

Using the fact that

$$
\sum_{n=0}^{N-1} x^{n}=\frac{1-x^{N}}{1-x}
$$

with $x=e^{-\left(\beta \Delta t-\frac{2 \pi i k}{N}\right)}$, the DFT is found to be

$$
F_{k}=\frac{1-e^{-\beta N \Delta t} e^{2 \pi i k}}{1-e^{-\beta N \Delta t} \cos \omega_{k} \Delta t-i e^{-\beta N \Delta t} \sin \omega_{k} \Delta t} .
$$

In the numerator, $e^{2 \pi i k}=\left(e^{\pi i}\right)^{2 k}=1$. The expression can be set into standard form as follows:

$$
\begin{aligned}
F_{k}= & \frac{1-e^{-\beta N \Delta t}}{1-e^{-\beta N \Delta t} \cos \omega_{k} \Delta t-i e^{-\beta N \Delta t} \sin \omega_{k} \Delta t} \\
& \cdot \frac{1-e^{-\beta N \Delta t} \cos \omega_{k} \Delta t+i e^{-\beta N \Delta t} \sin \omega_{k} \Delta t}{1-e^{-\beta N \Delta t} \cos \omega_{k} \Delta t+i e^{-\beta N \Delta t} \sin \omega_{k} \Delta t} \\
= & \frac{1-e^{-\beta \Delta t} \cos \omega_{k} \Delta t-e^{-\beta N \Delta t}+e^{-\beta(N+1) \Delta t} \cos \omega_{k} \Delta t}{1-2 e^{-\beta \Delta t} \cos \omega_{k} \Delta t+e^{-2 \beta \Delta t}} \\
& +i \frac{\left(e^{-\beta \Delta t}-e^{-\beta(N+1) \Delta t}\right) \sin \omega_{k} \Delta t}{1-2 e^{-\beta \Delta t} \cos \omega_{k} \Delta t+e^{-2 \beta \Delta t}} .
\end{aligned}
$$

The ratio of the real part of $F_{k}$ to the imaginary part is therefore

$$
\begin{aligned}
& \frac{\operatorname{Re}\left(F_{k}\right)}{\operatorname{Im}\left(F_{k}\right)} \\
& \quad=\frac{1-e^{-\beta N \Delta t}-e^{-\beta \Delta t} \cos \omega_{k} \Delta t+e^{-\beta(N+1) \Delta t} \cos \omega_{k} \Delta t}{e^{-\beta \Delta t} \sin \omega_{k} \Delta t-e^{-\beta(N+1) \Delta t} \sin \omega_{k} \Delta t} .
\end{aligned}
$$

This expression is of the form $-a x^{N+1}+x^{N}+a x-1=0$, in which $x=e^{-\beta \Delta t}$ and

$$
a=\frac{\operatorname{Re}\left(F_{k}\right)}{\operatorname{Im}\left(F_{k}\right)} \sin \omega_{k} \Delta t+\cos \omega_{k} \Delta t .
$$

This polynomial factors to $\left(x^{N}-1\right)(-a x+1)=0$. The first term can only be zero if $\beta, N$, or $\Delta t$ are zero. Therefore, for any nontrivial case,

$$
\left[\frac{\operatorname{Re}\left(F_{k}\right)}{\operatorname{Im}\left(F_{k}\right)} \sin \omega_{k} \Delta t+\cos \omega_{k} \Delta t\right] e^{-\beta \Delta t}=1,
$$

which can be solved for $\beta$ to give Eq. (9). Moreover, it is not difficult to show that the same expression is obtained for a waveform with a nonzero $y$ offset and a pre-exponential factor other than unity.

\section{APPENDIX C: DETERMINATION OF $\boldsymbol{\beta}$ FROM SUCCESSIVE INTEGRATION}

If the exponentially decaying waveform is

$$
f_{i}=A e^{-\beta t_{i}}+b,
$$

where $t_{i}=i \Delta t$ for data points equally spaced in time, the integral in Eq. (7) is approximated as the direct sum

$$
\begin{aligned}
& I_{n}=\sum_{k=0}^{n} f_{k} \Delta t=A \sum_{k=0}^{n} e^{-\beta k \Delta t} \Delta t+b(n+1) \Delta t \\
& =A \Delta t\left(\frac{1-e^{-\beta \Delta t} e^{-\beta t_{n}}}{1-e^{-\beta \Delta t}}\right)+b t_{n}+b \Delta t,
\end{aligned}
$$

where we have used

$$
\sum_{k=0}^{N} x^{k}=\frac{1-x^{N+1}}{1-x} \text {. }
$$

We now use Eq. (C1) to replace $e^{-\beta t_{n}}$ in Eq. (C3) for $I_{n}$ with $\left(f_{n}-b\right) / A$ obtaining

$$
I_{n}=\frac{A \Delta t}{1-e^{-\beta \Delta t}}+b \Delta t-\frac{e^{-\beta \Delta t}}{1-e^{-\beta \Delta t}}\left(f_{n}-b\right) \Delta t+b t_{n},
$$

which can be solved for $f_{n}$, yielding

$$
f_{n}=\frac{A+b}{e^{-\beta \Delta t}}-\frac{1-e^{-\beta \Delta t}}{e^{-\beta \Delta t} \Delta t} I_{n}+\frac{\left(1-e^{-\beta \Delta t}\right)}{e^{-\beta \Delta t} \Delta t} b t_{n} .
$$

This equation is in the form of Eq. (5) and the coefficients can be determined by solving Eq. (6). The coefficients are identified as

$$
\begin{aligned}
& a_{0}=\frac{A+b}{e^{-\beta \Delta t}}, \\
& a_{1}=-\frac{1-e^{-\beta \Delta t}}{e^{-\beta \Delta t} \Delta t}, \\
& a_{2}=\frac{\left(1-e^{-\beta \Delta t}\right)}{e^{-\beta \Delta t} \Delta t} b .
\end{aligned}
$$

Therefore, the coefficients of the exponentially decaying waveform are as follows:

$$
\begin{aligned}
& \beta=\frac{1}{\Delta t} \ln \left(1-a_{1} \Delta t\right), \\
& b=-\frac{a_{2}}{a_{1}}, \\
& A=a_{0} e^{-\beta \Delta t}-b .
\end{aligned}
$$

\section{APPENDIX D: DETERMINATION OF $\tau$ FROM THE PHASE OF THE FFT}

The value of the Fourier transform at a particular frequency $\omega$ may be written as 


$$
F(\omega)=A(\omega) e^{i \phi(\omega)},
$$

where $A$ is the amplitude and $\phi$ is the phase of the Fourier component at frequency $\omega$. The real and imaginary parts can be determined by writing the exponential as

$$
F(\omega)=A(\omega)[\cos \phi(\omega)+i \sin \phi(\omega)] .
$$

The amplitude cancels in the ratio of the real to the imaginary

$$
\frac{\operatorname{Re}[F(\omega)]}{\operatorname{Im}[F(\omega)]}=\frac{\cos \phi(\omega)}{\sin \phi(\omega)}=\cot \phi(\omega) .
$$

Therefore, for the determination of $\beta$ in Eq. (9), only the phase of the FFT is required.

${ }^{1}$ P. D. Kirchner, W. J. Schaff, G. N. Maracas, L. F. Eastman, T. I. Chappell, and C. M. Ransom, J. Appl. Phys. 52, 6462 (1981).

${ }^{2}$ M. Mazurenka, R. Wada, A. J. L. Shillings, T. J. A. Butler, J. M. Beames, and A. J. Orr-Ewing, Appl. Phys. B: Lasers Opt. B81, 135 (2005).

${ }^{3}$ T. J. A. Butler, J. L. Miller, and A. J. Orr-Ewing, J. Chem. Phys. 126, 174302 (2007)

${ }^{4}$ M. Mazurenka, L. Wilkins, J. V. Macpherson, P. R. Unwin, and S. R. Mackenzie, Anal. Chem. 78, 6833 (2006)

${ }^{5}$ R. Wada and A. J. Orr-Ewing, Analyst (Cambridge, U.K.) 130, 1595 (2005).

${ }^{6}$ R. Wada, J. M. Beames, and A. J. Orr-Ewing, J. Atmos. Chem. 58, 69 (2007).

${ }^{7}$ D. Halmer, G. von Basum, P. Hering, and M. Murtz, Rev. Sci. Instrum. 75, 2187 (2004).

${ }^{8}$ I. Matheson, Anal. Instrum. (N. Y.) 16, 345 (1987).

${ }^{9}$ K. L. Bechtel, R. N. Zare, A. A. Kachanov, S. S. Sanders, and B. A.
Paldus, Anal. Chem. 77, 1177 (2005).

${ }^{10}$ D. Halmer, G. von Basum, M. Horstjann, P. Hering, and M. Murtz, Isotopes Environ. Health Stud. 41, 303 (2005).

${ }^{11}$ D. Halmer, G. von Basum, P. Hering, and M. Murtz, Opt. Lett. 30, 2314 (2005).

${ }^{12}$ D. Halmer, S. Thelen, P. Hering, and M. Murtz, Appl. Phys. B: Lasers Opt. B85, 437 (2006).

${ }^{13}$ J. Hargrove, L. M. Wang, K. Muyskens, M. Muyskens, D. Medina, S. Zaide, and J. S. Zhang, Environ. Sci. Technol. 40, 7868 (2006).

${ }^{14}$ J. T. Hodges and D. Lisak, Appl. Phys. B: Lasers Opt. B85, 375 (2006).

${ }^{15}$ D. Marinov, J. M. Rey, M. G. Muller, and M. W. Sigrist, Appl. Opt. 46, 3981 (2007).

${ }^{16}$ V. Motto-Ros, J. Morville, and P. Rairoux, Appl. Phys. B: Lasers Opt. 87, 531 (2007).

${ }^{17}$ M. Murtz, D. Halmer, M. Horstjann, S. Thelen, and P. Hering, Spectrochim. Acta, Part A 63, 963 (2006).

${ }^{18}$ A. K. Y. Ngai, S. T. Persijn, G. Von Basum, and F. J. M. Harren, Appl. Phys. B: Lasers Opt. B85, 173 (2006).

${ }^{19}$ S. Stry, S. Thelen, J. Sacher, D. Halmer, P. Hering, and M. Murtz, Appl. Phys. B: Lasers Opt. B85, 365 (2006).

${ }^{20}$ H. Verbraak, A. K. Y. Ngai, S. T. Persijn, F. J. M. Harren, and H. Linnartz, Chem. Phys. Lett. 442, 145 (2007).

${ }^{21}$ D. E. Vogler and M. W. Sigrist, Appl. Phys. B: Lasers Opt. 85, 349 (2006).

${ }^{22}$ E. H. Wahl, B. Fidric, C. W. Rella, S. Koulikov, B. Kharlamov, S. Tan, A. A. Kachanov, B. A. Richman, E. R. Crosson, B. A. Paldus, S. Kalaskar, and D. R. Bowling, Isotopes Environ. Health Stud. 42, 21 (2006).

${ }^{23}$ T. G. Spence, C. C. Harb, B. A. Paldus, R. N. Zare, B. Willke, and R. L. Byer, Rev. Sci. Instrum. 71, 347 (2000).

${ }^{24}$ J. L. Miller and A. J. Orr-Ewing, J. Chem. Phys. 126, 174303 (2007).

${ }^{25}$ T. J. A. Butler, J. L. Miller, and A. J. Orr-Ewing, J. Chem. Phys. 126, 174302 (2007).

${ }^{26}$ R. Engeln, G. von Helden, G. Berden, and G. Meijer, Chem. Phys. Lett. 262, 105 (1996). 